\title{
THE
}

\section{One-Dimensional Model Systems: Theoretical Survey}

Jill C. Bonner

University of Rhode Island

Follow this and additional works at: https://digitalcommons.uri.edu/phys_facpubs

Terms of Use

All rights reserved under copyright.

\section{Citation/Publisher Attribution}

Jill C. Bonner. One-Dimensional Model Systems: Theoretical Survey. J. Appl. Phys. 49 (1978), 1299.

Available at: http://dx.doi.org/10.1063/1.325026

This Article is brought to you for free and open access by the Physics at DigitalCommons@URI. It has been accepted for inclusion in Physics Faculty Publications by an authorized administrator of DigitalCommons@URI. For more information, please contact digitalcommons-group@uri.edu. 


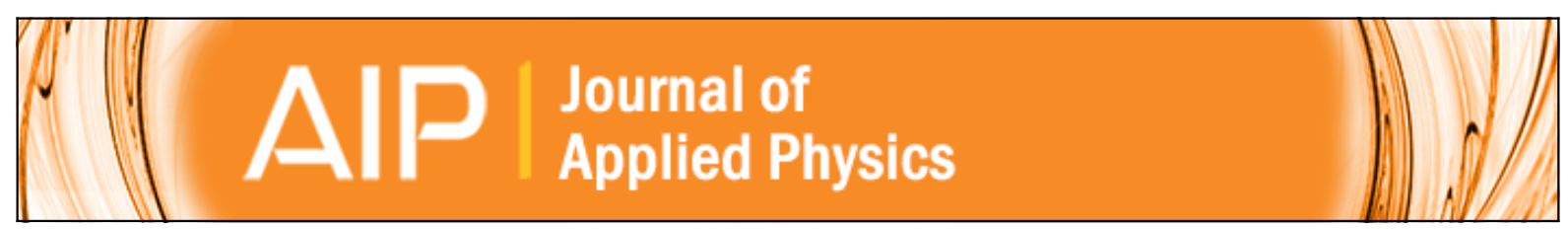

\section{One-dimensional model systems: Theoretical survey}

Jill C. Bonner

Citation: Journal of Applied Physics 49, 1299 (1978); doi: 10.1063/1.325026

View online: http://dx.doi.org/10.1063/1.325026

View Table of Contents: http://scitation.aip.org/content/aip/journal/jap/49/3?ver=pdfcov

Published by the AIP Publishing

\section{Articles you may be interested in}

One-dimensional and quasi-one-dimensional electron systems in nanochannels

Low Temp. Phys. 32, 86 (2006); 10.1063/1.2161934

Spin populations in one-dimensional alternant spin systems: A theoretical approach

J. Chem. Phys. 109, 5770 (1998); 10.1063/1.477199

Considerations about equivalent models in a classical one-dimensional system

Am. J. Phys. 48, 1088 (1980); 10.1119/1.12293

One-dimensional models for two-electron systems

Am. J. Phys. 43, 790 (1975); 10.1119/1.9708

One-Dimensional Plasma Model

Phys. Fluids 5, 445 (1962); 10.1063/1.1706638

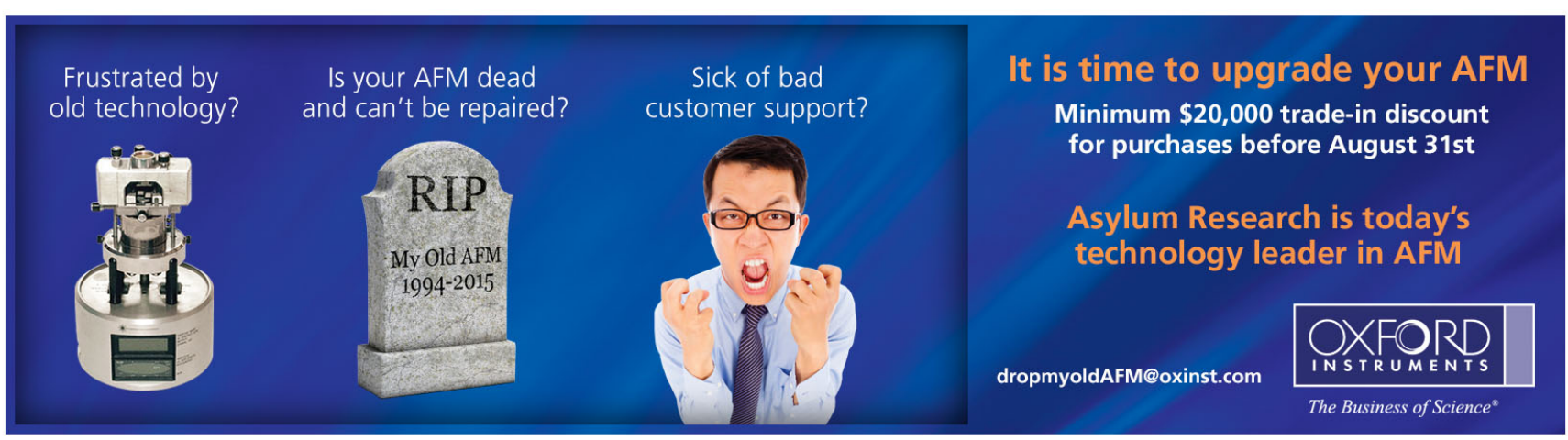


TUTORIAL SESSION

ONE-DIMENSIONAL MODEL SYSTEMS: THEORETICAL SURVEY*

Jil1 C. Bonner

Physies Department, Unfversity of Rhode Island Kingston, RI 02881

\section{ABSTRACT}

In the early $1960^{\prime} s$, one dimensional model systems were regarded as amusing toys with the advantage of being far more easily solvable than their "real" threedimensional counterparts. Now essentially 1-D (quasi1-D) magnets can be "tailor-made" in the laboratory. Even more popular is the field of organic conductors like TTF. TCNQ, which are naturally quas1-1-D. Current1y solitons and related solutions of non-linear, dispersive 1-D differential equations are ubiquitous in physics, including the area of 1-D magnetism. These developments are discussed in the Introduction. The rest of this paper is concerned with model Hamiltonians, model comparisons, critical singularities in 1-D (quasi-1-D) systems, accuracy of numerical techniques in comparison with exact solutions, brief accounts of dilute and disordered 1-D systems, and 1-D spin dynamics. Finally, a comment is made on a variety of interesting isomorphisms between I-D magnets and phenomena in several other areas of physics, for example 2-D ferroelectrics, fieldtheoretic models, and realistic fluids. Comparison of theory and experiment has been the subject of several excellent reviews and is therefore not discussed here.

\section{INTRODUCTION}

The Ising model of cooperative magnetism was suggested by Lenz and solved in one-dimension (1-D) by Ising in 1925 [1]. The solution was essentially complete as a function of both temperature, $T$, and applied magnetic field, H. Later work has discussed in some detail the correlation functions [2]. In 1944, a very famous solution of the two-dimenstonal (2-D) Ising model by Onsager appeared [1]. The solution provided information on the thermal properties in zero-field only: A complete solution, including $\mathrm{H} \neq 0$, is lacking to this day. Again, considerable additional work has been done on the correlation functions [3]. The 1-D Ising model is interesting as a soluble, non-trivial N-body problem and widespread and ingenfous exploitation of the 2-D Ising model solution has given tremendous impetus to the developing field of critical phenomena $[3,4]$. Unfortunately, the Ising model has its Iimitations as a model for magnetic phenomena. It is a purely classical, as opposed to a quantum-mechanical, model, and is therefore tnadequate to describe both static and dynamic phenomena. Good experimental examples of Ising model magnets are rare.

In the early 1960's an important sequence of solutions of a variety of 1-D magnetic insulator models appeared. Fisher was able to demonstrate a remarkably simple analytic solution for the ciassical (spin $S=\infty$ ) linear chain in zero field. The solution yielded both thermal properties and correlation functions [5]. The 1-D XY model with spin $S=1 / 2$ was solved analytically, independently by Lieb, Schultz and Mattis, with emphasis on the correlation functions, and by Katsura, with emphasis on the thermal and magnetic properties [6]. The Ratsura solution included also exact results for the 1-D transverse Ising model. An analytic solution for the $S=1 / 2$ Heisenberg linear chain is not available even now, though important progress has been made over the past few years. Therefore, Bonner and Fisher [7] and, Independently Griffiths, developed a technique of numerical extrapolation of exact results for a sequence of finite Heisenberg chains of increasing $N$ to the $\mathrm{N}=\infty \lim \mathrm{t}$. In this way an extensive set of results for $\mathrm{a} 11 \mathrm{H}$ and $\mathrm{T}$ and some results for the $\mathrm{T}=0$ correlation functions were obtained with accuracy comparable to experiment. Other important results for the $S=1 / 2$ Helsenberg antiferromagnet $1 c$ ( $A F)$ chain were an analyt1c calculation by des Clolzeaux and Pearson of the
R. ALBEN, CHAIRPERSON

lowest excited states (spin-wave triplets) and an analytic result for the zero-point susceptibility by Griffiths [8]. The relevance of these 1-D solutions to real experimental systems was demonstrated for the first time by Griffiths [ 9 ] in conjunction with workers at the Kamerlingh Onnes Laboratorium, Leiden. Interest in 1-D magnets among experimentalists and theorists was stimulated by this result and, particularly, by an annotated collection of reprinted papers on a variety of 1-D model systems, including lattice gases, dynamical disordered crystal lattices, many-fermion gases (electron gases) as well as magnets. The collection appeared in book form, and remains today an important and influential introduction to $1-D$ theory [10].

In the early $1960^{\prime}$ s 1-D model systems were interesting for a variety of reasons, most of which remain in force today. First of all, an essentially complete, exact or accurate numerical, solution could be obtained in one dimension, whereas the corresponding 3-D problem was hopelessly intractable. The enormous difficulty in 3-D naturally led to the development of a variety of approximate techniques for problems in manybody cooperative phenomena. These included mean-field theory, Bethe, Bethe-Peterls-Weiss and higher-order cluster approximations, the random phase approximation and higher-order Green's function calculations, and spin-wave theory. The 1-D results provided a testing ground for such general approximate techniques, and it is remarkable how inaccurate they are for, say, the 1-D Helsenberg antiferromagnet. The failure is not only quantitative but even qualitative. To be fair, there are reasons to argue that the inalequacies of the above theorles are most pronounced for low dimensionality and low spin value. Nevertheless, more caution than is often the case should perhaps be exercised in relation to the use of theories of the above type. very recently the renormalization group (RG) approach in the position space formulation has been tested and evaluated on $1-D$ spin-1/2 Ising models and spin-1 Ising models (showing $\mathrm{T}_{\mathrm{c}}=0$ tricritical phenomena) [11]. Considerably greater difficulty is experienced in applying RG theory to quantum-mechanical systems (for example, the $S=1 / 2$ Heisemberg modeI or $\mathrm{XY}$ mode1) [12] than to classical systems (Ising model or $S=\infty$ Heisenberg model). The Q.M. models are not only of interest in their own right, especially the $2-D$ cases [13], but also as models of quark confinement [12]. The study of 1-D quantum systems might well aid the development of an effective RG approach.

In some magnetic crystals, 1-D magnetic interaction pathways occur naturally between metallic ions as a consequence of magnetic structure (e.g. copper tetramine sulfate, $\left.\mathrm{Cu}\left(\mathrm{NH}_{3}\right)_{4} \mathrm{SO}_{4} \mathrm{H}_{2} \mathrm{O}, \mathrm{CTS}^{\gamma}[14\}\right)$; special alignment of orbitals (dy orbitals of $\mathrm{Cu}^{2+}$ in $\mathrm{KCuF} 3$ [14]); or between lonic components of magnetic organic complexes ( $\pi$-orbltal interactions in $\mathrm{TTF}^{+} \mathrm{PLS}_{4} \mathrm{C}_{4}\left(\mathrm{CF}_{3}\right)_{4}$, TTF BDT( $P t)$ [17]). Now it is possible to grow 1-D magnets "to order" by organic spacer techniques. For example, $\mathrm{CoCl}_{2} \cdot 2 \mathrm{H}_{2} \mathrm{O}$ is a "poor" $1-\mathrm{D}$ magnet. If, however, the $\mathrm{H}_{2} \mathrm{O}$ molecules are replaced by large organic pyridines to give $\mathrm{CoCl}_{2} \cdot 2 \mathrm{NC}_{5} \mathrm{H}_{5}$, the $\mathrm{Co}^{2+}$ lon chains are pushed much further apart, and the 1-D character is enhanced [14]. Consequently, over the past few years there has been considerable activity in finding "good" experimental 1-D magnets, and a wide assortment is now ava11able. This sttuation $1 \mathrm{~s}$ especially interesting to coordination chemists. Since the theory of superexchange remains on a far from secure footing, chemists are tending to investigate empirically the vartation of exchange constant with bond length and bond angle using families of 1-D magnets, relying on the complete and accurate statistical mechanical knowledge not available in higher-D systems. One important source of uncertainty in the processing of the data is thus removed.

An important area of current hot pursutt in $1-\mathrm{D}$ physics is the field of quasi-1-D organic conductors, semiconductors, and even insulators [15]. Materials 11ke the famous TTF.TCNQ are called organic metals because of their high conductivity at low temperature and its negat1ve temperature coefficlent. They are plent1- 
ful and nacurally anisotropic, 1.e. quasi-1-D, unlike the 1-D magnets which of cen have to be specially "engineered" $[14]$. Considerable interest, and also controversy, was stimulated by a prediction of Little that organic conductors might show "high temperature" superconductivity at, say, $50 \mathrm{~K}$. The discovery recently of superconductivity at $0.27 \mathrm{k}$ in polysulfur nitride (SN) unique in having no metallic components, boosted activity in this area. The technological importance of a $50 \mathrm{~K}$ superconductor, or even "good" organic conductors and photoconductors, is, of course, immense. Unfortunately, there is an Inherent lattice instability in 1-D systems, the famous Peierls transition [16], which distorts the underlying lattice so as to induce a phase tramsition from "metallic" to insulating (semiconducting) behavior as the temperature is lowered. Clearly, Peierls transitions are both interesting and technologically undestrable, for they compece with the putative superconducting transition and act to suppress $i t$. Recently, the insulating analogue of the Peierls transition has been discovered in some members of a class of quasi-1-D metallo-organic complex compounds, bisdithiolenes with TTF [17]. In this transition, an insulator-insulator transition, a unfform 1-i AF Heisenberg chain with a single exchange constant $J$, undergoes a dimerization at the spin-Peierls transition temperature to an alternating $A F$ Heisenberg chain. That is, pairs of spins move respectively closer and further apart and the 1-D magnetic system has two alternating exchange constants $J_{1}, J_{2}$, with $J_{1}>J_{2}$, say. AccordIng to careful numerical calculations on the alternatIng chain [18], a magnetic energy gap opens up between the singlet ground state and the lowest excited states (triplet excitors) for al1 $J_{1} \neq J_{2}$. The existence of such a gap is cructal to spin-Pelerls theory and also to the extensive fleld of exciton theory in physical chemistry [19]. Since the alternating linear chain Hamtltonian still possesses complete isotropic spin symmetry, doubts have been raised, based on classical (spin $\infty$ ) 1-D systems and approximate techniques, about the existence of such a gap [20], but the numerical evidence for it is strong [18].

It appears that 1-D physics is likely to exert its special fascination for some time to come. As fast as one area of interest attains a degree of matarity, e.g. 1-D magnetic insulators, a new area takes over, e.g., the fleld of organic conductors and $1-D$ metals [15]. Recently the solfton phenomenon has become very fashionable in all areas of physics, not just solid state physics. Solitons, kinks, and solitary travel1 ing wave phenomena are special solutions of $1-D$ differential equations which have the characteristics of being both non-linear and dispersive. Solitons have the unusual property that after they interact, they proceed on their way with shape and velocity essentially unchanged. Solitons have turned up in particle and fleld theorles, water waves, plasmas, SQUID theory, charge and spin density waves in 1-D conductors, and displacfve ferroelectrics [21]. There is now reason to believe that solitong are characterlstic excitations in 1-D conrinum ferromagnets, both classical $(S=\infty)$ and quantum [22]. Solitons may also be fmportant in $1-D$ antiferromsgnets, in connection with spin-Peierls and generalised Peierls transitions.

other areas of solid state physics where exact 1-D solutions are appearing thick and fast include Fermi and Bose gas delta function models [23], the linear Hubbard model [24], a 1-D plasma which crystallises as a Wigner solid [25], and the La1-5utherland model which includes the Hubbard model and a dilute magnetic model as spectal cases [26].

While the major emphasis of this review concerns 1-i) magnetic insulators, it should be noted that there has been considerable recent progress in solving 1-D electron gas models stimulated by interest in 1-D organic electrical conductors noted above. The electron gases include both continuum and lattice versions, and theories can incorporate the effects of spin and backward scattering [27]. An important equivalence between a $1-D$ electron gas and a 2-D coulomb gas has been noted [28]. These solutions are informative about charge and spin density wave phenomena.

Returning to $1-D$ magnetic insulators, the model solutions of the early 1960's have been augmented by the following important recent developments. Blume and co-workers have extended the solution of the $S=\infty$ Heisenberg linear chaln to include a non-zero magnetic ffeld by numerically based transfer matrix techniques [29]. The quantum, $S=1 / 2$ Heisenberg model has been solved exactly in a continuum (as opposed to discrete lattice) version on the basis of techniques for solving the 1-D many-fermion Tomonaga-Luttinger wodels [30]. A solution for the $S=1 \mathrm{XY}$ continum model has also appeared [13a], and essentially exact calculations on $1-D$ Ising models for $S>I / 2$ have been developed [31]. An interesting sequence of 1-D "n-vector" models has been solved (in zero field) by Stanley [32]. The number of spin-comonents is denoted by $n: n=1$ is the spin $1 / 2$ Ising model, $\mathrm{n}=2$ is the classical ( $\operatorname{spln} \infty$ ) $\mathrm{XY}$ model, $n=3$ is the classical Heisenberg model and, finally $\mathrm{n}=\infty$ gives the Berlin-Kac spherical model. Extension can be made to include biquadrattc as well as bilinear spin interactions [33]. The numerical extrapolation approach of Bonner and Fisher has been applied ro a variety of 1-D models where the possibility of exact solutions seems remote. Examples are the 1-D Hubbard model [34] (an extenston to the alternating version would be of interest), the Heisenberg ladder model suggested to describe copper nitrate [35], and Heisenberg-cype models for $S>1 / 2$, including single-ion anfsotropy [36].

To complete the Introduction, it seems appropriate to review the present status of exact, analytic results for the spin $1 / 2$ Ising-Heisenberg-XY linear chain. The mathematical analysis required for this $1-D$ model is definitely more complicated than that required to solve the $2-D$ Ising model. Since the $2-D$ Ising solution has been so fruitful in the development of the modern theorles of critical phenomena, it would seem profitable to pursue the $1-D$ model solution in equivalent detall. Early analytic work beginning in the 1930's has been revilewed by Thompson [2], A breakthrough by Yang and Yang about 1966 [37] resulted in a detailed solution as a function of anisotropy and magnetic field for $T=0$ only. However, this was $8 u f f i c-$ ient to determine the character of the interesting critical singularities. The finite temperature properties presented a greater problem. Progress has been made by Johnson and McCoy [38], and Independently by Takahashi [39], on the basis of a formulation due to Gaudin [40]. An Important paper by Johnson, Krinsky and McCoy deals with the elementary excitations of the general XYZ mode1 [41]. Nevertheless, information is still most notably lacking for the cases of the Heisenberg ferroand antiferromagnet, and the Ising-Heisenberg ferromagnet for $k=0$.

\section{MAGNETIC MODEL SOLUTIONS}

The effective spin Hamiltonian on which attention is primarily focussed in this review is

$$
H=-2 J \sum_{i=1}^{N}\left\{a s_{1}^{z} s_{i+1}^{z}+b S_{i}^{x} S_{i+1} x_{+} c s_{i}^{y} s_{i+1}^{y}\right\}
$$

We have bilinear spin coupling, assume nearest-neighbor spin interactions only, and the effective exchange constant is $J$ modified for the spin couplings in different directions by factors $a, b$ and $c$. The Ising model is obtained by putting $b=c=0$ : (In general by letting any two of $a, b, c$ equal zero). The $x Y$ model is obtalned by putting $a=0$ : (In general by letting any one of $a, b, c$ equal zero). If $a=b=c$, we have complete rotational symetry in spin space and obtain the Hefsenberg model which may, of course, be written in vector form

$$
H=-2 J_{1} \sum_{1}^{N} \vec{s}_{1} \cdot \vec{s}_{1+1}
$$

if $\mathrm{J}>0$, the spins may lower their energy by aligning paralle 1 and we have a Heisenberg ferromagnet. If $\mathrm{J}<0$, the antiparallel spin alignment is favored and we have 
an antiferromagnet. If $a, b$ and $c$ are not equal spin anisotropy prevalls. If $a>b \simeq c$, we have uniaxial (easy axis) anisotropy. On the other hand, if $a<b \approx c$, planar (easy plane) anisotropy results. The magnitude and type of anlsotropy in the effective spin Hamiltonian results from crystal fleld effects, as noted, for example, by de Jongh [14]. The effects of an applied magnetic field can be represented by the addition of a Zeeman term $-g \beta \overrightarrow{\mathrm{H}} \cdot \sum_{i} \overrightarrow{\mathrm{S}}_{i}$ to the Hamiltonian (1). Crystal

fleld effects may be accounted for by a term $\Delta \Sigma\left(S_{1}^{z}\right)^{2}$. Finally, the spin value may range over $1 / 2 \leq S \leq \infty$. The static properties commonly measured by experimentalists are the initial (zero-field) susceptibility, $X$, the specific heat at constant field, $\mathrm{C}_{H}$, and the magnetization isotherms as a function of applied field, $M_{T}$ vs. H. Theoretical information, which is essentially complete for the major magnetic models Ising, Heisenberg and $X Y$, is now avallable for compartson with experiment as a function both of model type and spin value. In addition, the effects of magnetic dimensionality are now reasonably well understood, qualitatively and quantitatively. These three major factors, dimensionality, spin, and model vartation, will be reviewed briefly, for their value in identifying the magnetic model underlying experimental results on (preferably more than) one of the static properties.

Concerning magnetic dimensionality, the characteristics of (quasi-) 1-D magnets are relatively low, broad maxima In the magnetic specific heat, and also in the AF and perpendicular susceptibilities. These maxima do not correspond to cooperative long-range-order (LRO) transitions, since for short-range (e.g., nearestneighbor) forces $T_{c}=0$ for ideal $1-D$ systems, and $T_{C}$ is relatively small for "good" 1-D experimental systems. The broad maxima therefore correspond to short-rangeorder (SRO). More speciflc details are available In previous articles and reviews. $[2,14,42,43]$. However, we note that the best method of determining magnetic dimensionality, pioneered by Shirane at Brookhaven, is quasi-elastic neutron scattering. Scattering in reciprocal space shows dramatic dimensionality effects, occuring at points for $3-D$ systems, lines for $2-D$ systems and planes for $1-D$ systems.

Information on spin variation is relatively recent. In general, the magnetic thermodynamic properties show a steady progressive variation in, say, the height and position of the rounded maximum as $S$ varies from $1 / 2$ to $\infty$. Results for the Ising parallel susceptibility are available [31], and for the Heisenberg susceptibility, which owing to spin-flop effects has a perpendicular character $[7,44]$. (The susceptibility parallel to the field direction vanishes as $T \rightarrow 0$ for uniaxially anisotropic AFs. Susceptibilities measured in a direction perpendicular to the easy axis show a non-zero value, $X_{0}$, as $\left.T \rightarrow 0.\right)$ Work of B18te [36] gives information on the spin variation of $\mathrm{C}_{\mathrm{H}}$, and Blote has in addition estimated the $T=0$ magnetization isotherms [45]. The spin variation is not very striking, except in one important respect. The $\mathrm{S}=\infty$ curves all show non-physical features at low temperatures. For example, the classical Heisenberg specific heat rises monotonically to a non-zero value as $\mathrm{T} \rightarrow 0$, instead of vanishing in accordance with the third law of thermodynamics, as do the curves for $S<\infty$.

Finally the model dependence of the $S=1 / 2 \mathrm{mag}-$ nets will be discussed. In Fig. 1 we see the perpendicular susceptibility for the Ising model, and $X$ for the XY model and Heisenberg AF. All three curves show qualitatively similar features. Fig. 2 shows. the zero-field specific heats. Note that the Ising and XY model specIf lc heats are independent of the sign of J. (This is also true of the $S=\infty$ Heisenberg model, not shown.) The $S=1 / 2$ Helsenberg model, however, shows pronounced differences between $F$ and AF. Fig. 3 displays the $T=0$ magnetization curves, $M_{T}$ vs $H$, for various models, and model dependent differences become significant. For the Ising model, the curve is a step function. For the Heisenberg AF, the curve starts out linearly (with slope= $x_{0}$ ) and saturates quadratically with field [7]. The critical

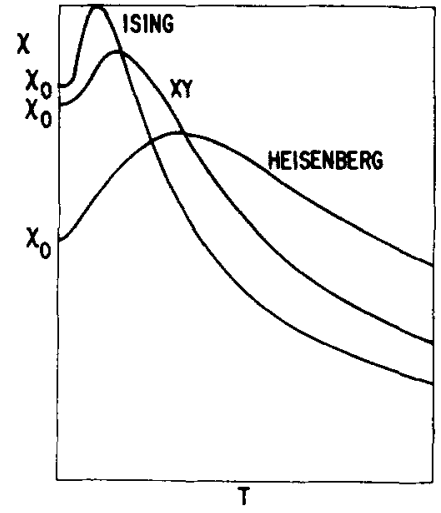

F1g. 1. Perpendicular susceptibilities of various $S=1 / 2$ $1-D$ magnets. The $\chi$ scale has been normalized.

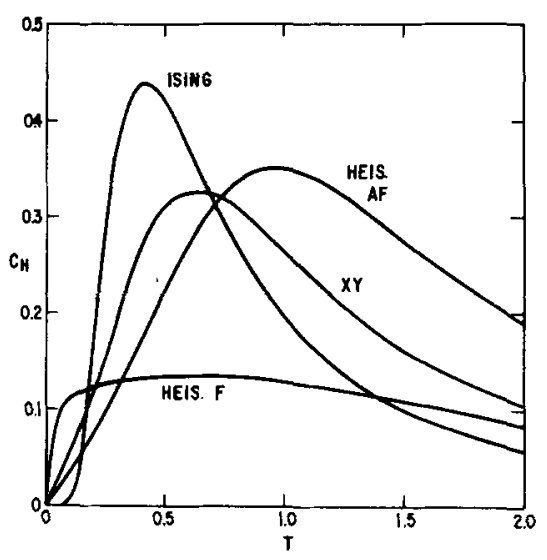

Fig. 2. Zero-field specific heats of various $S=1 / 2$ $1-D$ magnets.

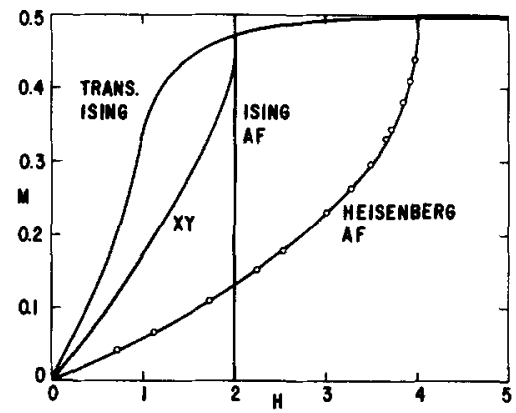

Fig. 3. $T=0$ magnetization curves vs. applied magnetic field for various $S=1 / 2$ magnets.

field is twice the Ising value. The $X Y$ curve is similar in shape to the Heisenberg model, but the critical field is the same as that for the Ising model. By contrast we show an Interesting curve for the Ising chain in a transverse fleld. Saturation is only attained for $\mathrm{H} \rightarrow \infty$, but a critical fleld is present as an inflection point, such that the field derivative (the zero-point suscept 1 bility) diverges logarithmically on each side. This is the same critical behavior as the zero-fleld specific heat of the 2-D regular Ising model, and illustrates a general result. This result strongly suggests that the critical singularities in a $\mathrm{D}$-dimensional mode 1 for $\mathrm{T}=0$, $H \neq 0$ should be the same as those for a $(D+1)-d i m e n-$ sional model for $H=0, T \neq 0$ [46]. More detailed discussions of model dependences are available $[7,14,43]$. One point of difference between the $1-D$ Ising model, and both $X Y$ and Helsenberg models, is the behavior of the correlation length $\xi(T)$. This quantity is a measure of the degree of correlation (i.e. degree of 
spin alignment) of spins along the chain. $\xi$ diverges as $T \rightarrow 0$, corresponding to a $T_{c}=0$ critical point. How ever, for the Ising model $\xi$ diverges exponentially fast (corresponding to relatively more rapid growth of correlation), and more slowly as $\mathrm{T}^{-1}$ for the $\mathrm{S}=\infty \mathrm{XY}$ and Heisenberg chains[53]. Quasi-elastic neutron scattering has provided experimental demonstration of the behavior of $\xi(\mathrm{T})$ [47]. The nature of the divergence of $\xi(\mathrm{T})$ for the $\mathrm{S}=1 / 2$ Heisenberg chain is not, as yet, known exactly.

\section{CRITICAL SINGULARITIES IN 1-D MODELS}

It is commonly sald that $3-D$ systems show $T_{C}>0$ phase transitions whereas $1-D$ systems show no phase transitions (and, by implication, are therefore less interesting). It should be noted that higher-D systems may have $T_{c}$ depressed by the presence of competing interactions. In fact, exactly solvable models do exist with no phase transition (even at $\mathrm{T}=0$ ) in zero field. Transitions occur only in non-zero magnetic fields [48]. Most 1-D systems with short-range forces show interesting critical singularities situated at $T_{c}=0$. However, even for 1-D models, if the magnetic interactions become sufficiently long-ranged, then $\mathrm{T}_{c}>0$. (In the limt of infinitely weak, infintte range interactions, (equivalent neighbor model), the critical behavior is mean-fieldlike). An important paper of Joyce $[49,50]$ gives analytic solutions for the long-range spherical model in both 1-D and higher D. In 1-D, 2-D and 3-D, three distinct regimes appear. For interaction range less than a special value, the critical behavior has the nearestneighbor character. For interaction range greater than a second, larger, special value, the critical behavior is mean-field-1ike. For intermediate range values, the critical exponents vary continuously between short-range and long-range limits. Similar behavior has been observed in numerical studies on the 1-D long-range Ising model [51] and in renormalization group treatments of the 3-D long-range Ising model [52].

It might be noted that for $\mathrm{T}_{\mathrm{C}}=0$ critical systems, hyperscaling relations may be formulated which are somewhat different from the usual relations, because factors of $\mathrm{T}$ and $\mathrm{T}^{2}$ in the basic definitions of thermodynamic properties give rise to confluent singularties at the $\mathrm{T}_{\mathrm{C}}=0$ critical point $[53]$.

\section{ACCURACY OF NUMERICAL TECHNIQUES}

Since analytic treatments are not always possible, even in $1-D$, it is interesting to evaluate the accuracy of numerical extrapolation methods described in the introduction. Fig. 4 is a comparison of numerical estimates of the zero-field $\mathrm{C}_{\mathrm{H}}$ for intermediate anisotropy $b=c=a / 2$, based on rings of up to 10 spins [43], with numerical solutions of coupled non-linear integral equations resulting from an analytic treatment of

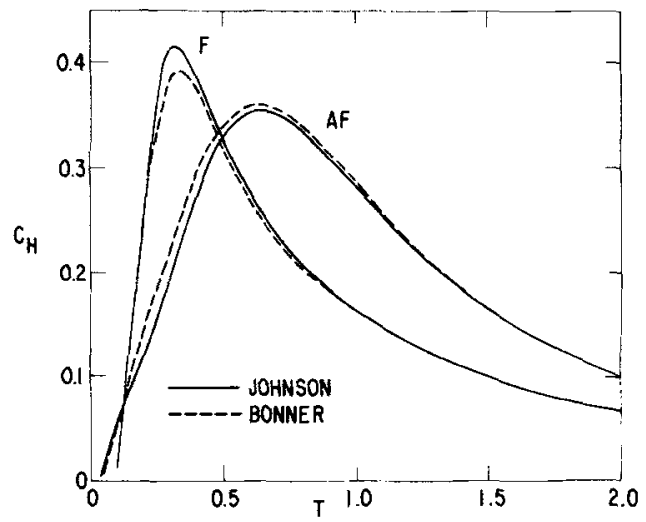

Fig. 4. Comparison of zero-field specific heats resulting from theoretical work and numerical extrapolations, for the anisotropic $S=1 / 2$ chain.

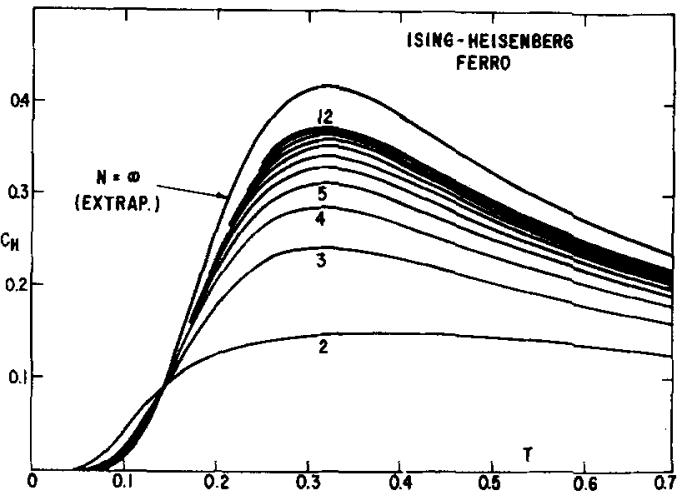

Fig. 5. Numerical extrapolations of Blote for the $S=1 / 2$ anisotropic 1inear ferromagnet.

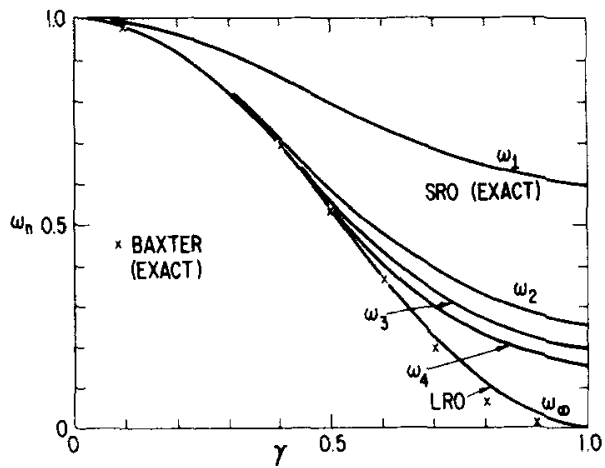

Fig. 6. Comparison of numerical extrapolations with exact analytic work for the $T=0$ LRO of the $1-D$ anisotropic antiferromagnet. Other pair correlation function ( $\omega_{n}(\gamma) \simeq\left\langle S_{0} S_{n}\right\rangle$ ) extrapolations are shown also. For $b=c$, the uniaxial anisotropy parameter is defined to be $\gamma=b / a$.

Johnson [38b]. Uncertainty is present in both methods. increasing as $T \neq 0$. The agreement, however, is generally satisfactory, discrepancies being comparable with experimental error. More recent calculations of Blote [54] for the anisotropic ferromagnet for free-ended chains of up to 12 spins are given in Fig. 5 and 111 strate the convergence of the finite systems to the extrapolated $\mathrm{N} \rightarrow \infty$ limit. The Blote extrapolation agrees with more refined recent calculations of Johnson [54] down to the lowest temperatures.

Two important recent analytic results for the Heisenberg $1-D$ AF are not generally well-known. The AF specific heat vanishes linearly with $\mathrm{T}$ as $\mathrm{T} \rightarrow 0$, with a coefficient numerically estimated to be 0.35 [7], and now exactly known to be 1/3 [39]. The long-range order parameter (LRO) for the Ising-Helsenberg AF was estimated numerically to vauish oniy at the Heisenberg limit. This prediction is confirmed by an exact result due to Baxter [55], and discrepancies between extrapolation and analytic result as a function of uniaxial anisotropy are reasonably small ( $\sim 5-10 \%$ at most), as shown in Fig. 6 .

\section{QUASI-1-D SYSTEMS}

In "real" 1-D systems, weak inter-chain interactions ultimately result in a $3-D$ cooperative ordering anomaly, situated at a lower temperature than the characteristic $1-D$ rounded maximum in a "good" example. The only available exact result illustrating this phenomenon is the we11-known work of Onsager $[1,14]$ for an Ising 2-D net with unequal "horizontal" and "vertica1" exchange constants. Many approximate theories have attempted to locate $\mathrm{T}_{c}$ for a specific model and ratio of inter-chain to intra-chain coupling constants, but quantitative a- 
greement among different approaches is not good [56]. A phenomenon recently observed experimentally in a serles of quasi-1-D $\mathrm{AF}^{\prime} \mathrm{s}$, where the phase transition initially increases in $T$ with increasing applied field, in contradiction to the predictions of mean-field theory, can be interpreted in connection with work of Kosterlitz, Nelson and Fisher [57]. Essentially, as soon as $H>0$, the quasi-1-D Heisenberg $A F$ behaves as a quasi-1-D XY model. Since $X Y$ ordering temperatures are relatively higher than Heisenberg ordering temperatures, the increase is qualitatively explained.

\section{DISORDERED AND DILUTE 1-D MAGNETS}

One-dimensional topology, or, equivalently, the low 1-D coordination number, emphasize strongly the effects of impurity and disorder in $1-D$ relative to higher $D$, where interaction pathways around the impurity are avai1 able. This feature, plus the much greater ease of solvabtlity in $1-D$, have made $1-D$ systems a happy hunting ground for researchers in the highly popular and growing area of dilute and disordered systems. The volume of interesting articles is already too vast for any realistic attempt at review in this short paper. The subject appears ripe for review on its own: unfortunately no such review is presently available, to the author's knowledge. Brief mention might be made of a comprehensive series of papers on $1-D$ solvable magnets with a distribution of exchange constants [58]; approximate treatments of quasi1-D systems with impurities [59]; exact solutions for randomly dilute Ising chalns and the $X Y$ chain with a random applied field [60]; exact calculations for random and impure classical Heisenberg chains [61]; and Monte Carlo studies on Ising spin glasses [62]. Work of Richards has represented a significant contribution to a correspondingly large body of experimental work [63].

\section{SPIN DYNAMICS OF QUASI-1-D SYSTEMS}

Theoretical study of dynamical properties is considerably more difficult than studies on static properties. Much work has been done, but the subject generall is on a relatively insecure footing owing to a lack of exact solutions for testing approximate theories. Accordingly, there has been considerable activity in $1-D$, which has been extensively reviewed in articles by Richards [64], Hone and Richards [64] and Steiner, Villain and Windsor [65]. Considerable attention has been paid to the classical Heisenberg chain because of its relative ease of solubility [66].

Infinite temperature correlations have been studied by numerical approximation techniques based on work of Bonner and Fisher [7] and Baker [67] for Heisenberg and $X Y$ chains [68]. A conjecture on the Gaussian time-dependence of the XY auto-correlation function [68] has recently been confirmed by exact work. A particularly complete treatment of the $T=0$ dynamical spin correlations of the $X Y$ chain is now avallable [70]. At low temperatures, the spin dynamics of 1-D Heisenberg AF's is interesting. By contrast with the classical Heisenberg chain, where all spectral weight is concentrated

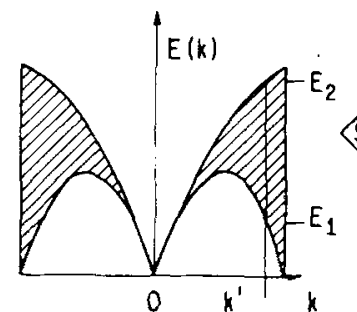

Fig. 7a. Two-parameter continuum excltations of the 1-D Heisenberg antiferromagnet. at the wel1-known double-sine (spin-wave) dispersion frequencies, numerical and approximate calculations [71,72] for the spin-1/2 Heisenberg AF indicate a "tail" of spectral weight above the des Cloizeaux and Pearson triplet spin-wave states. The situation is sketched in Fig. 7 .

Numerical [71] and analytical [41] studies indicate that the des Cloizeaux-Pearson states form the lower boundary of a two-parameter continuum (shown shaded) comprising degenerate singlet and triplet states. Numerical studies indicate that only the triplet states carry spectral weight, and also that non-negligible weight occurs at the top of the double continuum. If this theoretical prediction is confirmed by more refined neutron scattering experiments [73], then a further important difference between quantum $(S=1 / 2)$ and classical $(S=\infty)$ linear chains appears. This difference in the low temperature spin dynamics is sketched in Fig. $7 \mathrm{~b}$.

\section{1-D MAGNETS, 2-D FERROELECTRICS AND OTHER CORRESPONDENCES}

An important relation exists between the 1-D archetypical XYZ model and a class of 2-D hydrogen-bonded ferro- and antiferroelectrics, which has proved fruitful in the theory of cooperative phenomena. The isomorphism occurs because the transfer matrix for the 2-D problem commutes with the Hamiltonian matrix for a 1-D system. Hence the correlation functions in the two problems are related, and spin anisotropy in the 1-D magnet corresponds to temperature in the 2-D ferroelectrics. Specifically, the 2-D Rys antiferroelectric model corresponds to the Ising-Heisenberg AF, the 2-D Slater KDP ferroelectric corresponds to the Ising-Heisenberg ferromagnet, and the ice problem is isomorphous to a $1-D$ ferromagnet with anfsotropy intermediate between Heisenberg and $X Y$. Extensive further detail is provided in reviews by Li eb and Wu [74], Nagle [50] and Nagle and Bonner [75].

To conclude this review let us survey further interesting transcriptions between 1-D magnets and other physical phenomena. A we11-known correspondence between magnets and lattice gases ( $f l u i d s$ ) is utilized in exact calculations for a $1-D$ continuum cluster interaction fluid [76]. This transcription has also been used to transform a 1-D Ising AF with a Baker-Kac ferromagnetic tail [77] into continuum fluids [78] and lattice gases with extended repulsive hard cores [78], which show both a liquid-vapor phase line ending in a critical point and a solid-liquid phase line. In general, $1-D$ magnetic systems with competing long range interactions show multicritical behavior $[77,79]$. The behavior of dilute magnetic alloys near the percolation threshold has been related to essentially 1-D properties of selfavoiding walks [80] and exact solutions for 1-D site percolation problems show breakdown of universality [80]. Excitations of the $1-\mathrm{D}$ XYZ spin $1 / 2$ model are equivalent to those of the field-theoretic (one space and one time dimension) Thirring model with suitable relations between coupling constants [22a].

\section{ACKNOWLEDGEMENTS}

Great appreciation is expressed to the many people who sent samples of their recent work and valuable commentaries. Unfortunately, space restrictions forbid citing them by name, and has made reference selection a painful decision process with unavoldable, but regretable omissions.

\section{REFERENCES}

*Supported by NATO Scientific Research Programe.

1. E. Ising, Z.Phys., 31253 (1925). L. Onsager, Phys. Rev. 65, 117 (1944).

2. C.J.Thompson, Phase Transitions and Critical Phenomena, Vo1. 1, Eds. C. Domb and M.S.Green (Academic Press, London) 1972, Chap. 5.

3. B.M. McCoy and T.T.Wu, The Two-Dimensional Ising Model, (Harvard Univ. Press, Cambridge, 1973).

4. M.E. Fisher, Rept. Prog. Phys.,30 615 (1967). 
5. M.E. Fisher, Amer. J. Phys. 32, 343 (1964); T. Nakamura, J. Phys. Soc. Japan, 7264 (1952).

6. E. LIeb, et al., Ann. Phys. (N.Y.) 16407 (1961). S. Katsura, Phys. Rev. 127, 1508 (1962).

7. J.C. Bonner and M.E. Fisher, Phys. Rev. $135 \mathbf{4 6 4 0}$ (1964). R.B. Griffiths, unpub. repts. $(\overline{1961}, 1963)$.

8. J.des Cloizeaux and J.J.Pearson, Phys. Rev. 1282131 (1962). R.B. Griffiths, Phys. Rev. 133 A768 (1964).

9. R.B. Griffiths, Phys. Rev. 135 A659 (1964).

10. E.H. Ifieb and D.C. Mattis, Mathematical Physics in One Dimension (Acadenic Press, N.Y.) 1966.

11. D.R. Nelson and M.E. Fisher, Ann. Phys. (N.Y.) 91 226(1975). S.Krinsky and D.Furman, Phys.Rev.BIl 2602 $(1975)$.

12. J. Rogiers and D.D. Betts, Physica 85A 553 (1976). R.C. Brower et al., Phys. Rev. Lett. 38 1231(1977).

13a. A.Luther and D.J.Scalapino, Phys. Rev. B16 1153 (1977) . (b) J.V.José et al., Phys. Rev, B16 $1 \overline{217}$ (1977).

14. L.J. deJongh and A.R. Miedema, Adv. Phys. 23 1(1974) L.J. deJongh, J.App1.Phys., to be published.

15. See Low Dimensional Cooperative Phenomena, Ed. H.J. Keller, NATO Adv. Study Inst., (Plenum Press, N.Y.) 1975

16. R. E.Peferls, Quantum Theory of Solids, (Oxford Univ. Press, U.R.) 1955, p.108.

17. J.W. Bray et a1., Phys, Rev. Lett. 35744 (1975).I.S. Jacobs et a1. Phys. Rev. B14 3036 (1976). J.W. Bray and S.T. Chui, Ferroelectrics. In press.

18. J.C. Bonner and H. Blote, unpublished work. W. Duffy Jr., and K.P. Barr, Phys, Rev, 165647 (1968).

19. 2.G. Soos, Ann. Rev. Phys.Chem., 25 121(1974), and J. Chen. Phys. 464284 (1967).

20. M. Drawid and J.W. Halley, A.I.P. Conf.Proc. 34 208 (1976).

21. A.C. Scott, et al.,Proc. I.E.E.E. 61 1443(1973); A.R.Bishop et al., Phys.Rev. B14, 2966 (1976)

22a. A.Luther, Phys.Rev. BI4 2153 (1976);(b) J.Tjon and J.Wright, Phys.Rev.B15 3470(1977); N. Gupta and B. Sutherland, Phys.Rev. A14 1790(1976).

23. M. Takahashi, Progr. Theor.Phys. 461388 (1971); C.K. Lai, Phys. Rev. A8 2567 (1973).

24. M. Takahashi, Progr. Theor. Phys. $431619(1970)$ and ibid 501519 (1973). R.A. Bari, phys.Rev. B7 4318 $(1973)$.

25. B.Sutherland, Phys.Rev.Lett. 34 1083(1975), and 1bid 35185 (1975).

26. B.Sutherland, Phys.Rev. B12 3795 (1975).

27. A. Luther and V.J.Emery, Phys.Rev. Lett. 33589 (1974); V.J.Emery et a1., Phys. Rev. B13 1272 (1976); M.J. Rice et al., Phys.Rev.lett. 36432 (1976); V.J. Emery, Phys.Rev.Lett. 37107 (1976).

28. S.T. Chui and P.A.Lee, Phys.Rev.Lett. 35315 (1975).

29. M. Blume et al, Phys. Rev. B11 4483 (1975).

30. A. Luther and I. Peschel, Phys.Rev. B12 3908 (1975).

31. M. Suzuki et al., J.Math.Phys. 8124 (1967).

32. H.E. Stanley, Phys. Rev. 179570 (1969).

33. L.L. Liu and R.I. Joseph, Phys.Rev.Lett.26 1378(1971) M.F. Thorpe and M.Blume, Phys.Rev. B5 1961 (1972).

34. H. Shiba, Progr. Theor. Phys. 482171 (1972). H.Sh1ba and P.A.Pincus, Phys.Rev. B5 1966 (1972).

35. J.C. Bonner and S.A. Friedberg, A.I.P. Conf.Proc. 181311 (1973).

36. H.W.J. Blote, Physica $79 \mathrm{~B} 427$ (1975). T. deNeef, J. Phys. A7 L171 (1974).

37. C.N.Yang and C.P.Yang, Phys.Rev. 147303 (1966); 150 321,327 (1966); 151258 (1966)

38a. J.D.Johnson and B.M.McCoy, Phys.Rev. A6 1613 (1972). (b) J.D.Johnson, Phys.Rev. A9 1743 (1974).

39. M.Takahash1, Progr.Theor.Phys, 501519 (1973)

40. M. Gaudin, Phys. Rev. Lett. 261301 (1971).

41. J.D. Johnson et al., Phys.Rev. A8 2526 (1973).

42. C.Domb and A.R. Miedema, Progress in Low Temperature Physics, Vo1.4, edited by C.J.Gorter (North-Holland, Amsterdam), Chap. 6.

43. J.C. Bonner, Thesis, Univ. of London, 1968.

44. C.Y.Weng, Thesis, Carnegie-Mellon Untv. 1968.

45. J.J. Smit et a1., Colloques Int. CNRS 242, Phys. Sous Champs Magnét Iques Intenses, 253 (1974).

46. M. Suzuki, Progr.Theor.Phys. 461337 (1971).

47. M. Blume, Neutron Inelastic Scattering (Int. Atomic Energy Agency, Vlenua) 1972, p.529.
48. J.C. Bonner, Bull. Amer.Phys.Soc, 19305 (1974).

49. G.S.Joyce, Phys.Rev. 146349 (1966).

50. J.F. Nagle, Treatise on Solid State Chemistry, Vol.5 Ed. N. B. Hannay, (Plenum Press) 1975, Chap. 1.

51. J.F. Nagle and J.C. Bonner, J.Phys. C. 3352 (1970).

52. M.E. Fisher, S.Ma and B.G.Nickel, Phys.Rev.Lett. 29 917 (1972). M. Suzuki,Progr.Theor.Phys.49 424 (1973)

53. G.A. Baker, Jr., and J.C.Bonner, Phys.Rev. B12 3741 (1975).

54. H.W.J.Blöte, unpub. work; J.D.Johnson, unpub. work.

55. R.J.Baxter, J.Stat.Phys. 9145 (1973).

56. Y.Imry et a1., Phys.Rev B12 1978 (1975); L.J. de Jongh and H.E.Stanley, Phys.Rev.Lett. 36817 (1976); D.P.Landau and T.Graim, Proc. 13th IUPAP Conf. on Statistical Physics, Haifa, Israel (1977).

57. J.M.Kosterlitz et al., Phys.Rev. B13 412 (1976); Y. Shapira and N.F.olivelra, Jr., Proc. 23rd Ann. MM Conf., Minneapolis, Minn. (1977).

58. G.Theodorou and M.H.Cohen, Phys.Rev.Lett. 371014 (1976), and related work.

59. D.Hone et a1., Phys.Rev. B12 $514 I$ (1975) and related work.

60. G.Fan and B.M.McCoy, Phys.Rev. 182614 (1969); M. Wortis, Phys.Rev. B10 4665 (1974); G.Grinstein et a1., Phys.Rev.Lett. $\underline{36} 1508$ (1976); E.R.Smith, J. phys. C3 1419 (1970).

61. T.Tonegawa et al., Phys.Rev. B11 4683 (1975).

62. D.P.Landau and M.Blume, Phys.Rev. B13 287 (1976).

63. P.M.Richards, Phys.Rev. B14 1239 (1976).

64. P.M.Richards, Proc. Int. School of Physics Enrico Fermi, Ed. K.A.Muller (North Holland, Amsterdam) 1973 p. 539. D.W.Hone and P.M.Richards, Ann. Rev. Mat. Science, 4337 (1974).

65. M. Steiner et a1., Adv. Phys. 2587 (1976).

66. P.Heller and M.Blume, Phys.Rev.Lett. 39962 (1977); G. Reiter and A.Sjölander, to be published; N.A. Lurie et a1., Phys. Rev. B9 2171 (1974); F.B.McLean and M.Blume, Phys, Rev. B7 1149 (1973).

67. G.A.Baker,Jr., et a1., Phys.Rev. 135 A1273 (1964).

68. A.Sur and I.J.Lowe, Phys.Rev. B1l 1980 (1975); A. Sur et al., Phys.Rev. Bl2 3845 (1975).

69. H.W.Capel and J.H.H.Perk, Phystea 87A 211 (1977).

70. H.G.Vaidya and C.A.Tracy, to be published.

71. J.C.Bonner et a1., A.I.P. Conf. Proc. 24335 (1974).

72. T.Todani and K.Kawasaki, Progr. Theor.Phys. 501216 (1973); P.C.Hohenberg and W.F.Brinkman, Phys.Rev. B10 128 (1974).

73. Y.Endoh et al., Phys. Rev.Lett. 32170 (1974).

74. E.H.L1eb and F.Y.Wu, Phase Transitions and Critical Phenomena, Vol. 1, Eds. C.Domb and M.S.Green (Academic Press, London) 1972, Chap. 8.

75. J.F.Nagle and J.C.Bonner, Ann.Rev.Phys.Chem. 27 291 (1976).

76. M.E.Fisher and B.U.Felderhof, Ann.Phys. $\underline{58} 176$ (1970).

77. J.F.Nagle, Phys.Rev. A2 2124 (1970).

78. P.Hemmer and G.Ste11, Phys.Rev.Lett. 241284 (1970); G.Stell and P.C.Hemmer, J.Chem.Phys. $\overline{56} 4274$ (1972); G.Stell et al., Phys.Rev.Lett. 28 292 (1972).

79. J.C.Bonner and J.F.Nagle, J.App1.Phys. 421280 (1971); J.F.Nagle and J.C.Bonner, J.Chem.Phys. 54729 (1971).

80. H.E.Stanley et a1., J.Phys. C 9 L553 (1976); P.J. Reynolds et al., J.Phys. A 10 L202 (1977); W.Klein et al., J.Phys. A to be published. 